\title{
Fatores de risco para ansiedade e depressão na gestação: Revisão sistemática de artigos empíricos
}

\author{
Amanda Kliemann* \\ Elisangela Böing** \\ Maria Aparecida Crepaldi***
}

\section{Resumo}

O objetivo deste estudo foi caracterizar a produção de artigos que mensurassem sintomas de ansiedade e depressão na gestação. Foram analisados quarenta e um artigos, nacionais e internacionais, publicados entre 2010 e 2016 em revistas indexadas nas bases Scielo, BVS-PSI, LILACS, CAPES, Pubmed e APA PsycNet. A maioria dos estudos era do tipo transversal e todos utilizaram como método o levantamento de dados. Como técnica de coleta de dados foram utilizadas escalas, questionários, inventários e entrevistas. Os fatores de risco para ansiedade e depressão encontrados são apresentados a partir de categorias temáticas. Ressalta-se a relevância do presente estudo para ações de prevenção em saúde mental no contexto pré-natal. Sugere-se que estudos futuros avancem na utilização de instrumentos psicométricos que considerem sintomas específicos da gestação e investiguem a presença de indicadores emocionais na mãe e no pai. Sugere-se a integração da metodologia quantitativa e qualitativa e estudos longitudinais para aprofundar a temática.

Palavras-chave: gravidez, ansiedade, depressão, fatores de risco.

\section{Factors for anxiety and depression during pregnancy: Systematic review of empiric articles}

\begin{abstract}
This study aims at characterizing the production of articles that measure symptoms of anxiety and depression during pregnancy. Forty-one national and international articles, published from 2010 to 2016 in journals indexed in the databases Scielo, BVS-PSI, LILACS, CAPES, Pubmed and APA PsycNet, were analyzed. Most of the studies used a cross-sectional approach and all of them used the data survey method. As data collect technic, scales, questionnaires, inventories and interviews were used. The found risk factors for anxiety and depression are presented through thematic categories. It is important to emphasize the relevance of the current study for the prevention actions in mental health in the prenatal context. It is recommended that future studies take further steps in the utilization of psychometric tools that take into consideration specific symptoms of pregnancy and research the presence of emotional indicators in the mother and the father. It is also recommended the inclusion of quantitative and qualitative methodology and longitudinal studies to deepen the subject.

Keywords: pregnancy, anxiety, depression, risk factors.
\end{abstract}

*Mestranda. Programa de Pós-Graduação em Psicologia, Centro de Filosofia e Ciências Humanas da Universidade Federal de Santa Catarina.
**Doutora. Programa de Pós-Graduação em Psicologia, Centro de Filosofia e Ciências Humanas da Universidade Federal de Santa Catarina.
***Doutora. Programa de Pós-Graduação em Psicologia, Centro de Filosofia e Ciências Humanas da Universidade Federal de Santa Catarina. 


\section{Introdução}

A gravidez enquanto momento de transição no ciclo de vida individual e familiar impõe uma série de reorganizações intrapsíquicas, familiares, de papéis sociais, socioeconômicas e profissionais (Bradt, 1995; Cêpeda, Brito \& Heitor, 2006; Maldonado, 2005) e sua vivência pode ser influenciada por diversos fatores como o contexto da mesma, a relação com o parceiro, recursos pessoais, história de vínculos afetivos e relações familiares, rede de apoio, entre outros (Bortoletti, 2007).

Por vivenciar a gestação em seu corpo, a mulher torna-se especialmente sensível e vulnerável emocionalmente. As profundas mudanças físicas, hormonais, psicológicas e de papéis sociais fazem com que a mulher vivencie um estado de regressão emocional, no qual se volta mais para si e para seu "mundo interno", o que se associa às memórias afetivas de sua própria infância e de suas relações familiares, especialmente da relação com a própria mãe. A condição de regressão emocional pode se expressar de diferentes formas, entre elas, através de labilidade emocional e da necessidade de receber afeto e suporte (Maldonado, 2005; Soifer, 1984). Além disso, a presença de um contexto de perdas e ganhos faz com que a gestação esteja invariavelmente ligada ao sentimento de ambivalência afetiva (Maldonado, 2005).

A gestação pode ser considerada um período de crise (Carter \& McGoldrick, 1995; Cerveny \& Berthoud, 2002; Langer, 1981; Maldonado, 2005; Soifer, 1984), envolvendo assim tensão e desequilíbrio, mas também a oportunidade de maturação e crescimento individual e familiar (Carter \& McGoldrick, 1995; Raphael-Leff, 1997). Dessa forma, a condição de maior vulnerabilidade emocional pode tanto aumentar a chance de desenvolvimento de transtornos emocionais, quanto gerar a oportunidade de resolução de conflitos psíquicos e de atingir novos níveis de integração, amadurecimento e expansão da personalidade (Klaus \& Kennel, 1992; Raphael-Leff, 1997).

Nesse sentido, é esperado que a mulher vivencie um certo grau de estresse, ansiedade e momentos de tristeza durante a gestação. Contudo, em algumas mulheres observa-se a exacerbação de sintomas de ansiedade, estresse e até mesmo a presença de quadros depressivos, o que se associa a uma maior probabilidade de complicações na gravidez, no parto e no puerpério e pode gerar repercussões negativas na saúde da mulher, do bebê e nas relações familiares (Maldonado, 2005).

Pesquisas têm indicado que a presença de estresse e de estados persistentes de ansiedade e depressão ao longo da gestação associam-se a desfechos obstétricos como o parto pré-termo, o baixo peso ao nascer e efeitos nocivos no desenvolvimento neurológico do feto, podendo ter efeitos futuros na regulação da atenção, desenvolvimento cognitivo e motor, temperamento e regulação emocional nos primeiros anos de vida da criança (Schetter \& Tanner, 2012). Ademais, a depressão na gestação tem sido considerada como um dos principais fatores de risco para depressão pós-parto, sendo, muitas vezes, uma continuação da depressão iniciada na gestação, o que pode gerar efeitos adversos no funcionamento psicossocial da mãe, na relação mãe-bebê e no desenvolvimento psicossocial da criança (Molina \& Kiely, 2011).

Dados epidemiológicos têm apontado que as prevalências de transtornos mentais comuns são semelhantes na gestação e no puerpério (Gavin, Gaynes, Lohr, Meltzer-Brody, Gartlehner \& Swinson, 2005), sendo o transtorno de ansiedade e a depressão os transtornos de maior prevalência durante a gravidez (Pereira \& Lovisi, 2008). Nesse sentido, o rastreamento de alterações emocionais durante a gestação constitui-se importante estratégia de prevenção em saúde mental e relacional para mãe-bebê-família.

A partir do exposto o objetivo do presente estudo foi a realização de uma revisão sistemática de artigos empíricos que mensuravam a ocorrência de ansiedade e depressão em gestantes e os fatores de risco associados.

\section{Método}

Foi realizada uma pesquisa bibliográfica em seis diferentes bases de dados no mês de maio de 2016, a saber: Scielo, BVS-PSI; LILACS, Portal da CAPES, Pubmed e APA PsycNet. Foram utilizadas seis estratégias de bus$\mathrm{ca}$, com os seguintes descritores em língua inglesa e os correspondentes em português. A escolha das palavras-chave baseou-se em consultas no catálogo de descritores do PubMed (MESH terms) e no sítio dos Descritores em Ciências da Saúde (DeCS), da BVS. A seguir as estratégias de busca que foram realizadas.

1. pregnancy AND (stress OR anxiety OR depression OR depressive);

2. pregnancy AND (stress OR anxiety OR depression OR depressive) AND prevalence;

3. pregnancy AND (stress OR anxiety OR depression OR depressive) AND "risk factors";

4. "high risk pregnancy" AND (stress OR anxiety OR depression OR depressive)

5. "high risk pregnancy" AND (stress OR anxiety OR depression OR depressive) AND prevalence; 
6. "high risk pregnancy" AND (stress OR anxiety OR depression OR depressive) AND "risk factors";

A análise do material ocorreu de acordo com as seguintes fases: (1) levantamento da produção científica nas bases de dados selecionadas; (2) exclusão dos materiais de acordo com os seguintes critérios de exclusão: aqueles que não fossem artigos empíricos; que não fossem em idioma inglês, português ou espanhol; que não correspondessem aos critérios referentes ao ano de publicação (2010 a 2016) e que não disponibilizassem o acesso ao texto completo; (3) leitura dos resumos e seleção dos artigos que apresentassem a mensuração da ocorrência de ansiedade, estresse e/ou depressão em gestantes; (4) exclusão de produções científicas que não apresentavam a utilização de instrumentos psicométricos para a mensuração de ansiedade, estresse e/ou depressão em gestantes; (5) importação do material selecionado para o software End Note X4, com o objetivo de realizar a retirada de trabalhos duplicados e avaliação dos resumos com base nos critérios previamente definidos; (6) leitura na íntegra de todos os artigos da amostra final $(\mathrm{N}=41)$ para apreciação das categorias de análise e seleção definitiva do material componente do corpo de trabalho.

Por fim, os artigos lidos integralmente foram classificados com relação a: nacionais ou internacionais, gestação de baixo ou alto risco, delineamento metodológico (tipo de estudo, método, técnica utilizada e análise de dados), variáveis estudadas e principais resultados relacionados aos fatores de risco para ansiedade e/ou depressão na gestação. É importante ressaltar que, como foram obtidos apenas dois estudos que avaliavam a variável estresse, optou-se por caracterizar os fatores de risco para ansiedade e/ou depressão na gestação encontrados nos estudos selecionados.

\section{Resultados}

O levantamento inicial de dados obteve 2333 resultados, destes: 28 no Scielo; 215 no BVS-PSI, 266 no Lilacs, 1083 no Pubmed, 547 no CAPES e 460 no APA PsycNet. Após a exclusão dos artigos, conforme os critérios apresentados, obteve-se um total de 41 artigos científicos, sendo: 01 do Scielo; 02 do BVS-PSI; 02 do Lilacs; 11 no Pubmed; 05 no CAPES e 20 do APA PsycNet, todos com acesso ao texto completo.

\section{Estudos Nacionais ou internacionais}

Dentre os estudos selecionados $80,48 \%$ são internacionais $(\mathrm{N}=33)$, enquanto que somente $6,43 \%(\mathrm{~N}=8)$ são nacionais.

\section{Contexto da gestação}

Um total de $71 \%$ dos estudos foi realizado no contexto de gestação de baixo risco $(\mathrm{N}=29)$, sendo assim, apenas 29\% eram referentes a gestações de alto risco $(\mathrm{N}=12)$.

\section{Delineamento metodológico}

A Tabela 1 apresenta os dados relativos aos tipos de estudos, métodos, técnicas utilizadas e análises de dados.

Tabela 1 - Tipos de estudos, métodos, técnicas utilizadas e análise de dados

\begin{tabular}{lc}
\hline Variável & $\mathrm{N}$ \\
\hline Tipo de estudo & 01 \\
Longitudinal & 40 \\
Transversal & 41 \\
Total & \\
Método & 41 \\
Levantamento de Dados & 41 \\
Total & \\
Técnica utilizada & 14 \\
Escalas & 17 \\
Escalas/Questionários & 5 \\
Escalas/Questionários/Entrevistas & 5 \\
Escalas/Inventários & 41 \\
Total & \\
Análise de dados & 41 \\
Quantitativa & 41 \\
Total & \\
Nota. n=frequência. &
\end{tabular}

A partir da análise da tabela 1 é possível observar que a maioria dos estudos seguiu o delineamento transversal, sendo apenas um longitudinal. Todos os estudos utilizaram o método de levantamento de dados. Além disso, a maioria dos trabalhos utilizou escalas e questionários para mensurar a ocorrência ou prevalência de ansiedade, estresse e depressão. A técnica de entrevista foi utilizada exclusivamente associada a outros instrumentos. Todos os trabalhos foram analisados quantitativamente.

Variáveis estudadas

Houve a predominância de estudos que mensuraram a variável depressão $(\mathrm{N}=29)$. Foram encontrados poucos estudos que mensuraram especificamente a ansiedade $(\mathrm{N}=2)$, sendo esta mais estudada em associação com a variável depressão $(\mathrm{N}=9)$. Além disso, foram encontrados apenas dois $(\mathrm{N}=2)$ estudos com foco na variável estresse, o que justificou que não fossem analisados os fatores de risco associados. 


\section{MARIA APARECIDA CREPALDI}

\section{Instrumentos psicométricos}

A Tabela 2 apresenta os instrumentos psicométricos utilizados nas pesquisas selecionadas, de acordo com cada variável mensurada.

Tabela 2 - Variáveis e instrumentos utilizados

\begin{tabular}{|c|c|c|}
\hline Variável & Instrumentos & $\mathrm{N}$ \\
\hline \multirow[t]{8}{*}{ Depressão } & Edinburgh Postnatal Depression Scalee (EPDS) & 18 \\
\hline & Center for Epidemiologic Studies Depression Scale (CES-D) & 3 \\
\hline & Hospital Anxiety and Depression Scale (HADS) & 2 \\
\hline & Patient Health Questionnaire (PHQ-9). & 2 \\
\hline & Hopkins Symptom Checklist-Depression Scale. & 1 \\
\hline & Chinese version of the Postpartum Depression Screen Scale (PDSS) & 1 \\
\hline & Self-rating Depression Scale & 1 \\
\hline & Total de Instrumentos Depressão & 13 \\
\hline \multirow[t]{7}{*}{ Ansiedade } & Spielberger State-Trait Anxiety & 2 \\
\hline & Inventory) & \\
\hline & Neuropsychiatric Interview & \\
\hline & Patient Health Questionnaire for Depression and Anxiety (PHQ-4) & 1 \\
\hline & Pregnancy-Related Anxiety Questionnaire Revised (PRAQ-R) & 1 \\
\hline & Depression Anxiety Stress Scale (DASS-21) & 1 \\
\hline & Total de Instrumentos Ansiedade & 8 \\
\hline \multirow[t]{3}{*}{ Estresse } & Depression Anxiety Stress Scale (DASS-21) & 1 \\
\hline & Prenatal Psychosocial Profile (PPP) & 1 \\
\hline & Total de Instrumentos Estresse Total Geral & 21 \\
\hline
\end{tabular}

Nota. $\mathrm{n}=$ frequência.

Em relação aos instrumentos é possível observar, na Tabela 2, a diversidade deles para medir a ocorrência de depressão ( $\mathrm{n}=11)$ entre os 38 estudos analisados, sendo o mais utilizado a Edinburgh Postnatal Depression Scale (EPDS) $(N=18)$. Para a mensuração de ocorrência de ansiedade foram identificados oito (08) instrumentos em 11 pesquisas diferentes, sendo o STAI A-trait scale e STAI-A-state scale os mais utilizados. Para a mensuração de ocorrência de estresse, foram encontrados 02 instrumentos, Depression Anxiety Stress Scale (DASS-21) e o Prenatal Psychosocial Profile (PPP).

\section{Fatores de Risco para ansiedade e depressão em gestantes}

Considerando que dos 41 artigos selecionados 38 avaliaram a presença de indicadores de depressão em gestantes, seja isoladamente $(\mathrm{N}=29)$, ou de forma associada a variável ansiedade ( $N=9$ ), optou-se por descrever os fatores de risco para depressão e para depressão e ansiedade de forma associada. Dessa forma, os fatores de risco foram inicialmente compilados a partir da leitura minuciosa dos estudos. A compilação foi organizada em dois grupos: fatores de risco para depressão e fatores de risco para depressão e ansiedade, de acordo com as variáveis envolvidas nas pesquisas. A partir dessa escolha, foram criadas categorias temáticas, de acordo com a similaridade entre as características dos trabalhos, a fim de organizar a apresentação dos resultados. Com a intenção de apresentar de modo integrado os resultados da categorização dos estudos foi organizado um mapa da literatura (Creswell, 2007), apresentado nas Figuras 1 e 2.

Em relação aos fatores de risco evidenciados nos artigos destacaram-se, por ordem de ocorrência: a) fatores socioeconômicos (15 estudos); b) histórico de saúde mental e complicações obstétricas (14 estudos cada); c) rede social de apoio frágil (13 estudos); d) nível educacional (11 estudos); e) idade materna (10 estudos); f) eventos estressores na gestação (09 estudos); g) uso de álcool, 


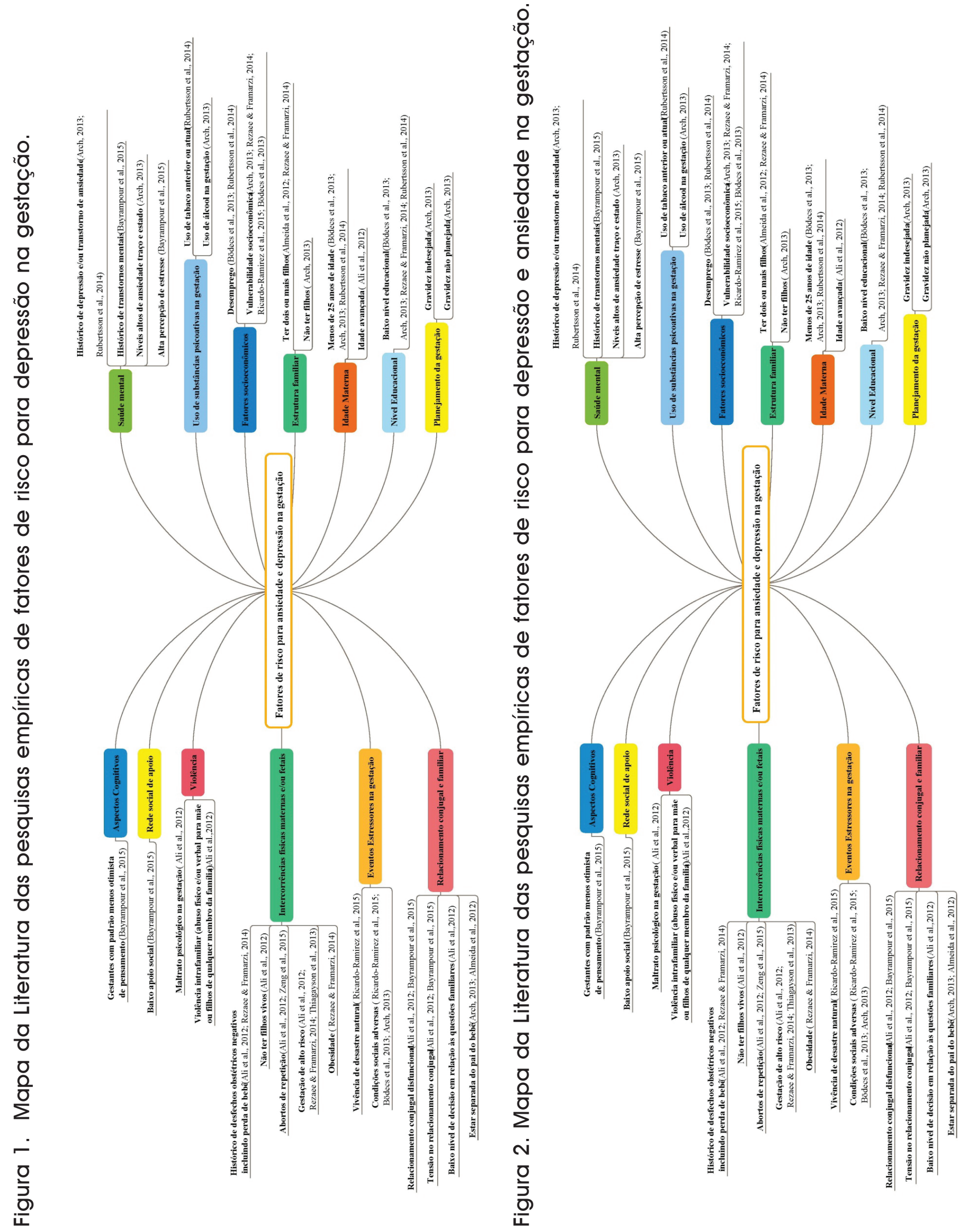


tabaco e/ou drogas ilícitas (05 estudos); h) violência (04 estudos); i) coping e aspectos cognitivos (01 estudo cada). Assim, percebe-se que os fatores socioeconômicos, o histórico de saúde mental, as complicações obstétricas e a rede de apoio frágil foram os principais fatores de risco para sintomas de depressão e ansiedade na gestação, em termos de frequência, nos estudos analisados.

\section{Discussão}

Apesar da crença socialmente difundida da gravidez como um período de plenitude e bem estar para as mulheres, os estudos indicam que os índices de ansiedade e depressão na gestação são altos (Almeida, Nunes, Camey, Pinheiro \& Schmidt, 2012; Benute, Nozzella, Prohaska, Liao, Lucia \& Zugaib, 2013; Gavin et al., 2005; Molina \& Kiely, 2011; Pereira \& Lovisi, 2008; Silva, Jansen, Souza, Moraes, Tomasi, Silva, Dias \& Pinheiro, 2010). Nesse sentido, as pesquisas analisadas reforçaram a necessidade de um olhar cuidadoso para os aspectos emocionais da mulher durante a gestação.

Os resultados da presente revisão evidenciaram ainda uma variedade de fatores que podem influenciar o estado psicológico materno durante a gestação, desde aspectos de atributos pessoais (idade, nível educacional, aspectos cognitivos e de coping), fisiológicos (complicações obstétricas), de histórico de saúde (histórico de saúde mental e uso de substâncias psicoativas), relacionais (estrutura familiar, relacionamento conjugal e familiar, violência e rede de apoio), contextuais (planejamento da gestação, eventos estressores e migração) a fatores que perpassam questões macrossociais como os fatores socioeconômicos, demonstrando o quanto a depressão e quadros de ansiedade são fenômenos complexos e multideterminados.

Em relação às pesquisas terem indicado a relação entre a mulher ser separada, não residir com o companheiro ou não ter apoio do mesmo com sintomatologia depressiva, para Molina e Kiely (2011) essa associação somente pode ser significativa se outros fatores de risco e mecanismos de proteção forem incluídos na análise como, por exemplo, o apoio da família e os recursos de enfrentamento da mulher. Nesse sentido, Dudas, Csatordai, Devosa, Toreki, Ando', Baraba's, Pa'1 e \& Kozinszky (2012) obtiveram como resultado que estar ou não casada não obteve diferença em relação aos índices de depressão, mas a qualidade da relação sim, já que relações instáveis correlacionaram-se com sintomas de depressão. Dessa forma, os autores ressaltaram que uma relação estável mesmo que o casal não esteja vivendo junto é mais protetiva do que um casamento com conflitos. Além disso, em locais em que não estar casada tem se tornado mais comum e encarado socialmente de forma positiva, essa condição torna-se menos associado a problemas de relacionamento e fator de risco para depressão gestacional (Dudas et al., 2012).

No que concerne a questões metodológicas, considerando a totalidade de utilização do delineamento quantitativo é possível pensar que com o objetivo de se analisar o impacto de certas variáveis na saúde mental materna em termos de frequência, prevalência e associações estatisticamente significativas se faz necessária a utilização de uma abordagem quantitativa. No entanto, a utilização de abordagens qualitativas visando uma compreensão individualizada, em que sejam aprofundadas questões subjetivas que influenciam os fenômenos estudados também é de suma relevância. Ressalta-se que nesse artigo parte-se do pressuposto de que tanto a abordagem quantitativa quanto a qualitativa possuem vantagens e limitações.

No presente estudo de revisão, foram considerados tanto aspectos metodológicos quanto os resultados das pesquisas. A partir disso, evidenciou-se a necessidade de se ampliar o foco das investigações e das discussões, procurando compreender a saúde mental materna desde os atributos pessoais (história de vida, personalidade, padrões de coping), considerando o contexto social e relacional (relação com figura materna, padrão de relações familiares intergeracional e recursos na comunidade), além de aspectos contextuais e culturais numa perspectiva macrossocial. Sugere-se ainda que estudos futuros investiguem a presença de indicadores emocionais na mãe e no pai, com o objetivo de ampliar o olhar para a díade coparental. Vale ressaltar que diferentes perspectivas teóricas podem contribuir para o estudo da psicologia perinatal.

A assistência pré-natal é peça primordial para a promoção da saúde mental materna e consequentemente infantil e familiar. No entanto, ainda nos dias de hoje, as ações de saúde no contexto do pré-natal são fortemente influenciadas pelo modelo biomédico, em que a saúde é considerada como a "ausência de doença" e a assistência é pautada no paradigma da ciência tradicional, isto é, da simplicidade, estabilidade e/ou previsibilidade e objetividade. Isso se reflete na abordagem que enfoca questões fisiológicas, desconsiderando fatores psicológicos, relacionais e dados do contexto da paciente. Dessa forma, salienta-se a importância de se tornar o cuidado pré-natal mais integral, sendo necessário sensibilizar as equipes 
de saúde para incluírem em sua rotina de pré-natal a investigação de aspectos de saúde mental e relacional e de conhecer e fortalecer a rede social de apoio da mulher gestante, além de outros aspectos psicossociais.

\section{Considerações finais}

Sugere-se a realização de pesquisas longitudinais para a compreensão das variáveis que influenciam a saúde mental materna no ciclo gravídico-puerperal e no decorrer do desenvolvimento da criança ao longo do tempo. Além disso, é necessário o avanço em termos de utilização de instrumentos específicos para a avaliação de indicadores emocionais no período gestacional, visto que de um total de 21 instrumentos utilizados nas pesquisas analisadas a maioria era validada para rastreio geral de ansiedade, estresse e depressão, com exceção de quatro instrumentos que consideravam a especificidade das manifestações psicológicas durante a gestação.

Os resultados da presente revisão reforçaram a necessidade de um olhar ampliado para se compreender os aspectos de saúde mental materna e prover um cuidado integral e efetivo para a mulher gestante e sua família. Por fim, ressalta-se a necessidade da mulher ser avaliada e amparada emocionalmente durante a gestação, e de receber apoio afetivo e efetivo da rede social, familiar e das equipes de saúde para que, assim, possa receber seu bebê e promover seu desenvolvimento.

\section{Referências}

Agostini, F., Neri, E., Salvatori, P., Dellabartola, S., Bozicevic, L. \& Monti, F. (2015). Antenatal depressive symptoms associated with specific life events and sources of social support among Italian women. Matern Child Health J., 19(5), 1131-41.

Akçalı Aslan P., Aydın, N., Yazıcı, E., Aksoy, A. N., Kirkan, T. S. \& Daloglu, G. A. (2014). Prevalence of depressive disorders and related factors in women in the first trimester of their pregnancies in Erzurum, Turkey. Int J Soc Psychiatry, 60(8), 809-17.

Ali, N. S., Azam, I. S., Ali, B. S., Tabbusum, G. \& Moin, S. S. (2012). Frequency and associated factors for anxiety and depression in pregnant women: a hospital-based cross-sectional study. Scientific World Journal., 1-9.

Almeida, M. S., Nunes, M. A., Camey, S., Pinheiro, A. P. \& Schmidt, M. I. (2012). Transtornos mentais em uma amostra de gestantes da rede de atenção básica de saúde no Sul do Brasil. Cad. Saúde Pública, Rio de Janeiro, 28(2), 385-393.

Arch, J. J. (2013). Pregnancy-specific anxiety: which women are highest and what are the alcohol-related risks? Comprehensive Psychiatry, 54, 217-228.

Bayrampour, H., McDonald, S. \& Tough, S. (2015). Risk factors of transient and persistent anxiety during pregnancy. Midwifery, 31, 582-589.

Benute, G. R. G., Nomura, R. M. Y., Reis, J. S., Junior, R. F., de Lucia, M. C. S. \& Zugaib, M. (2010). Depression during pregnancy in women with a medical disorder: risk factors and perinatal outcomes. Clinics, 65(11),1127-1131.

Benute, G. R., Nizzella, D. C., Prohaska, C., Liao, A., Lucia, M. C. \& Zugaib, M. (2013). Twin pregnancies: evaluation of major depression, stress and social support. Twin Res Hum Genet.,16(2), 629-33.

Bödecs, T., Szilágyi, E., Cholnoky, P., Sándor, J., Gonda, X., Rihmer, Z. \& Horváth, B. (2013). Prevalence and psychosocial background of anxiety and depression emerging during the first trimester of pregnancy: data from a hungarian population-based. Psychiatria Danubina, 25 (4), 352-358.

Bortoletti, F. F. (2007). Psicodinâmica do ciclo gravídico puerperal. In F. F. Bortoletti (Org.), Psicologia na prática obstétrica: abordagem interdisciplinar (pp. 21- 31). São Paulo: Manole.

Bradt, J. O. (1995). Tornando-se pais: Famílias com filhos pequenos. In Carter, E. \& McGoldrick, M. As mudanças no ciclo de vida familiar: Uma estrutura para a terapia familiar (pp. 206-221). Porto Alegre: Artmed.

Carolan-Olah, M. \& Barry, M. (2014). Antenatal stress: An Irish case study. Midwifery, 30, 310-316.

Carter, B., \& McGoldrick, M. (1995). As mudanças no ciclo de vida familiar: uma estrutura para a terapia familiar (2. ed.). Porto Alegre: Artes Médicas.

Cepêda, T., Brito, I. \& Heitor, M. J. (2006). Promoção da Saúde Mental na Gravidez e Primeira Infância. In Ministério da Saúde, Direcção-Geral da Saúde. Promoşão da Saúde Mental na Gravidez e Primeira Infancia: Manual de orientação para profissionais de saúde. Portugal. Retrieved from https://www. dgs.pt/documentos-e-publicacoes/promocao-da-saude-mental-na-gravidez-e-primeira-infancia-manual-de-orientacao-para-profissionais-de-saude.aspx

Cerveny, C. M. O. \& Berthoud, C. M. E. (2002). Visitando a família ao longo do ciclo vital. São Paulo: Casa do Psicólogo.

Coelho, F. M., Pinheiro, R. T., Silva, R. A., Quevedo, L. de Á., Souza, L. D., Castelli, R. D., ... Pinheiro, K. A. (2013) Major depressive disorder during teenage pregnancy: socio-demographic, obstetric and psychosocial correlates. Rev Bras Psiquiatr, 35(1), 51-6.

Creswell, J. W. (2007). Projeto de pesquisa - métodos qualitativo, quantitativo e misto. 2a. Ed. Porto Alegre: Artmed.

Dagklis, T., Papazisis, G., Tsakiridis, I., Chouliara, F., Mamopoulos, A. \& Rousso, D. (2016). Prevalence of antenatal depression and associated factors among pregnant women hospitalized in a high-risk pregnancy unit in Greece. Soc Psychiatry Psychiatr Epidemiol, 51:1025-1031.

Dibaba, Y., Fantahun, M. \& Hindin, M. J. (2013).The association of unwanted pregnancy and social support with depressive symptoms in pregnancy: evidence from rural Southwestern Ethiopia. BMC Pregnancy and Childbirth, 13:135.

Dong, X., Qu Z, L. F., Jiang, X., Wang, Y., Chui, C. H., Wang, X., ... Zhang, X. (2013). Depression and its risk factors among pregnant women in Sichuan earthquake area and non-earthquake struck area in China. J Affect Disord, 151(2), 566-72.

Dudas, R. B., Csatordai, S., Devosa, I., Toreki, A., Ando, B., Baraba, K., ... Kozinszky , Z. (2012). Obstetric and psychosocial risk factors for depressive symptoms during pregnancy. Psychiatry Research, 200, 323-328.

Fortner, R. T., Pekow, P., Dole, N., Markenson, G. \& Chasan-Taber, L. (2011). Risk factors for prenatal depressive symptoms among Hispanic women. Matern Child Health J.,15(8),1287-95.

Gavin, N. I., Gaynes, B. N., Lohr, K. N., Meltzer-Brody, S., Gartlehner, G. \& Swinson, T. Perinatal depression: a systematic review of prevalence and incidence. Obstet Gynecol, 106, 1071-83.

Giurgescu, C., Zenk, S. N., Templin, T. N., Engeland, C.G., Dancy, B. L., Park, C. G., ... Misra, D. P.(2015).The Impact of Neighborhood Environment, Social Support, and Avoidance Coping on Depressive Symptoms of Pregnant African-American Women. Womens Health Issues, 25(3), 294-302.

Goedhart, G., Snijders, A. C., Hesselink, A. E., Van Poppel, M. N., Bonsel, G. J. \& Vrijkotte, T. G. M. (2010). Maternal depressive symptoms in relation to perinatal mortality and morbity: results from a large multiethnic cohort study. Psychosom Med, 72, 769-776.

Juhas, T. R., Benute, G. R. G., Lucia, M. C. S., Francisco, R. P. V. (2014). Major depression in high-risk obstetric inpatients and outpatients. MedicalExpress, $1(2), 87-90$.

Klaus, M. \& Kennel, J. (1992). Pais/ bebê: a formação do apego. Porto Alegre: Artes Médicas.

Langer, M. (1981). Maternidade e Sexo. Porto Alegre: Artes Médicas.

Lau, Y., Yin, L. \& Wang, Y. (2011). Antenatal depressive symptomatology, family conflict and social support among Chengdu Chinese women. Matern Child Health J., 15(8), 1416-26.

Maldonado, M. T. (2005). Psicologia da Gravidez: (17 ed). São Paulo: Saraiva.

Miszkurka, M., Goulet, L. \& Zunzunegui, M. V. (2012). Antenatal depressive symptoms among Canadian-born and immigrant women in Quebec: differential exposure and vulnerability to contextual risk factors. Soc Psychiatry Psychiatr Epidemiol, 47(10),1639-48. 
Miyake, Y., Tanaka, K. \& Arakawa, M. (2012). Employment, income, and education and prevalence of depressive symptoms during pregnancy: the Kyushu Okinawa Maternal and Child Health Study. BMC Psychiatry, 12 (117).

Molina, K. M. \& Kiely, M. (2011). Understanding depressive symptoms among high-risk, pregnant, African-American women. Womens Health Issues, 21(4), 293-303.

Pereira, P. K., Lovisi, G. M., Lima, L. A. \& Legay, L. F. (2010). Complicações obstétricas, eventos estressantes, violência e depressão durante a gravidez em adolescentes atendidas em unidade básica de saúde. Archives of Clinical Psychiatry, 37(5), 216-222.

Raphael-Leff, J. (1997). Gravidez: a história interior. Porto Alegre: Editora Artes Médicas.

Rezaee, R. \& Framarzi, M. (2014). Predictors of mental health during pregnancy. Iran I Nurs Midwifery,19(7), 45-50.

Ricardo-Ramírez, C., Álvarez-Gómez, M., Ocampo-Saldarriaga, M. V. \& Tirado-Otálvaro, A. F. (2015). Prevalencia de tamizaje positivo para depresión y ansiedad en gestantes de alto riesgo obstétrico en una clínica de Medellín, entre enero y agosto de 2013. Factores de riesgo asociados. Revista Colombiana de Obstetricia y Ginecología, 66(2), 94-102.

Rochat, T. J.,Tomlinson, M., Newell, M. \& Stein, A. (2013). Detection of antenatal depression in rural HIV-affected populations with short and ultrashort versions of the Edinburgh Postnatal Depression Scale (EPDS). Arch Womens Ment Health, 16, 401-410.

Rodrigues, O. M. P. R. \& Schiavo, R. de A. (2011). Stress na gestação e no puerpério: uma correlação com a depressão pós-parto. Revista Brasileira de Ginecologia e Obstetrícia, 33(9), 252-257.

Roesch, S. C. Schetter, C. D. \& Woo, G. (2004). Modeling the types and timing of stress in pregnancy. Journal Anxiety, Stress \& Coping, 17, 187-102.

Rubertsson, C., Hellström, J., Cross, M. \& Sydsjö, G. (2014). Anxiety in early pregnancy: prevalence and contributing factors. Arch Womens Ment Health, 17, 221-228.

Rwakarema, M., Shahirose, S., Premji, E., Nyanza, C., Riziki, P. \& Palacios-Derflingher, L. (2015). Antenatal depression is associated with pregnancy-related anxiety, partner relations, and wealth in women in Northern Tanzania: a cross-sectional study. BMC Womens Health., 15 (68).

Schetter, D. C. \& Tanner, L. (2012). Anxiety, depression and stress in pregnancy: implications for mothers, children, research, and practice. Current Opinion in Psychiatry, 25(2), 141-148.

Senturk, V., Abas, M., Berksun, O. \& Stewart, R. (2011). Social support and antenatal depression in extended and nuclear family environments in Turkey: a cross-sectional survey. BMC Psychiatry, 11 (48).

Silva, R. A. da, Jansen, K., Souza, L. Dias de M., Moraes, I. G. da S., Tomasi, E., Silva, G. Del G., ... Pinheiro, R. T. (2010). Depression during pregnancy in the Brazilian public health care system. Revista Brasileira de Psiquiatria, 32(2), 139-144.
Soifer, R. (1984) Psicologia da gravidez, parto e puerpério. Porto Alegre: Artes Médicas.

Stewart, R. C., Umar, E., Tomenson, B. \& Creed, F. (2014). A cross-sectional study of antenatal depression and associated factors in Malawi. Arch Womens Ment Health, 17, 145-154.

Thiagayson, P., Krishnaswamy, G., Lim M. L., Sung, S. C., Haley, C. L., Fung, D. S., ... Chen, H. (2013). Depression and anxiety in Singaporean high-risk pregnancies - prevalence and screening. Gen Hosp Psychiatry, 35(2),112-6.

Thiengo, D. L., Pereira, P. K., Santos, J. F, de C., Cavalcanti, M. T. \& Lovisi, G. M. (2012). Depressão durante a gestação e os desfechos na saúde do recém-nascido: coorte de mães atendidas em unidade básica de saúde. Jornal Brasileiro de Psiquiatria, 61(4), 214-220.

Usuda, K., Nishi, D., Makino, M., Tachimori, H., Matsuoka, Y., Sano, Y. \& Konishi, T., ...Takeshima, T. (2016). Prevalence and related factors of common mental disorders during pregnancy in Japan: a cross-sectional study. Biopsychosoc Med., 10(17).

Verbeek, T., Arjadi, R., Vendrik, J. J., Burger, H. \& Berger, M. Y. (2015). Anxiety and depression during pregnancy in Central America: a cross-sectional study among pregnant women in the developing country Nicaragua. BMC Psychiatry, 15 (292)

Yanikkerem E., Ay S., Mutlu S. \& Goker A. (2013). Antenatal depression: prevalence and risk factors in a hospital based Turkish sample. I Pak Med Assoc, 63(4), 472-7.

Zahra, B. M. \& Elham, R. (2014). The Prevalence of Depression in Pregnant Women with Sleep Disorder. J Psychiatry, 17(6).

Zeng, Y., Cui, Y. \& Li, J. (2015). Prevalence and predictors of antenatal depressive symptoms among Chinese women in their third trimester: a cross-sectional survey. BMC Psychiatry, 15 (66).

Zhao, Y., Kane, I., Mao, L., Shi, S.,Wang, J., Lin, Q. \& Lu, L. (2016) The Prevalence of Antenatal Depression and its Related Factors in Chinese Pregnant Women who Present with Obstetrical Complications. Archives of Psychiatric Nursing ,30, 316-321.

Wangel, A. M., Schei, B., Ryding, E. L. \& Ostman, M. (2012). Mental health status in pregnancy among native and non-native Swedish-speaking women: a Bidens study. Acta Obstet Gynecol Scand, 91 (12), 1395-401.

Weobong, B., Soremekun, S., Augustinus, H. A., Asbroek, T., Amenga-Etego, S., Danso, S., ... Kirkwood, B. R. (2014). Prevalence and determinants of antenatal depression among pregnant women in a predominantly rural population in Ghana: The DON population-based study. Journal of Affective Disorders, 165, 1-7.

Submetido em: 22-4-2017

Aceito em: 1-8-2017 